\title{
Data-Independent Feature Learning with Markov Random Fields in Convolutional Neural Networks
}

DOI:

10.1016/j.neucom.2019.03.107

\section{Document Version}

Accepted author manuscript

Link to publication record in Manchester Research Explorer

\section{Citation for published version (APA):}

Peng, Y., Hankins, R., \& Yin, H. (2020). Data-Independent Feature Learning with Markov Random Fields in Convolutional Neural Networks. Neurocomputing, 24-35. https://doi.org/10.1016/j.neucom.2019.03.107

\section{Published in:}

Neurocomputing

\section{Citing this paper}

Please note that where the full-text provided on Manchester Research Explorer is the Author Accepted Manuscript or Proof version this may differ from the final Published version. If citing, it is advised that you check and use the publisher's definitive version.

\section{General rights}

Copyright and moral rights for the publications made accessible in the Research Explorer are retained by the authors and/or other copyright owners and it is a condition of accessing publications that users recognise and abide by the legal requirements associated with these rights.

\section{Takedown policy}

If you believe that this document breaches copyright please refer to the University of Manchester's Takedown Procedures [http://man.ac.uk/04Y6Bo] or contact uml.scholarlycommunications@manchester.ac.uk providing relevant details, so we can investigate your claim.

\section{OPEN ACCESS}




\title{
Data-Independent Feature Learning with Markov Random Fields in Convolutional Neural Networks
}

\author{
Yao Peng, Richard Hankins, Hujun Yin* \\ School of Electrical and Electronic Engineering, The University of Manchester, Manchester, M13 9PL, UK
}

\begin{abstract}
In image classification, deriving robust image representations is a key process that determines the performance of vision systems. Numerous image features and descriptors have been developed manually over the years. As an alternative, however, deep neural networks, in particular convolutional neural networks (CNNs), have become popular for learning image features or representations from data and have demonstrated remarkable performance in many real-world applications. But CNNs often require huge amount of labelled data, which may be prohibitive in many applications, as well as long training times. This paper considers an alternative, data-independent means of obtaining features for CNNs. The proposed framework makes use of the Markov random field (MRF) and self-organising map (SOM) to generate basic features and model both intra- and inter-image dependencies. Various MRF textures are synthesised first, and are then clustered by a convolutional translation-invariant SOM, to form generic image features. These features can be directly applied as early convolutional filters of the CNN, leading to a new way of deriving effective features for image classification. The MRF framework also offers a theoretical and transparent way to examine and determine the influence of image features on performance of CNNs. Comprehensive experiments on the MNIST, rotated MNIST, CIFAR-10 and CIFAR-100 datasets were conducted with results outperforming most state-of-the-art models of similar complexity.
\end{abstract}

Keywords: Convolutional neural networks, Image representation, Markov random fields, Gibbs distribution, Self-organising maps, Image classification, Image features.

\section{Introduction}

Image classification relies on learning and identifying discriminant features of objects or regions of interest in images. Applications have grown considerably over the ${ }_{25}$ 5 last few decades, from biometrics, object detection and recognition, to medicine and robotics. However, despite a great deal of effort, image classification based on visual content remains a challenging problem in computer vision, due to the almost infinite ways of representing images as ${ }_{30}$ 10 well as large intra-class variations. A common approach has been to design or handcraft a set of features that are invariant or robust to these variations while still remaining discriminatory for different classes. Numerous such image features have been proposed in the literature. Although low-level features can be carefully designed with great success for certain datasets and tasks, designing effective features for any new task requires prior knowledge and careful and extensive engineering, a process that is often empirical, ad hoc and time consuming. This has ${ }_{40}$

\footnotetext{
* Corresponding author

Email addresses: yao.peng@manchester.ac.uk (Yao Peng), richard.hankins@manchester.ac.uk (Richard Hankins), hujun.yin@manchester.ac.uk (Hujun Yin)
}

representations directly from raw data, especially by deep neural networks [1, 2.

Compared to handcrafted features and shallow neural network based methods, deep neural networks use more sophisticated structures, comprising multiple processing layers to build up robust local image representations with multiple levels of abstraction 3 . When applied to computer vision tasks, deep leaning methods substantially outperform conventional handcrafted approaches and shallow learning [4, 5, 6, 7, 8, 9, 10, 11, 12, 13, 14, 15, 16, 17, 18, 19, 20. As a key ingredient to the success of deep learning, convolutional neural networks (CNNs) possess a natural ability to learn large-scale invariance and deformations [21. Recent successes and advances have been witnessed in increasing number of applications of various image classification tasks.

Despite these remarkable successes and state-of-theart performances by CNNs (e.g. in handwritten digit, face recognition and image categorisation [22, 23, 24, 25, 26]), their feature learning mechanism and optimal configurations of their structures are not well understood 22. These networks are often limited or tuned to specified applications and may not generalise well to other image classification tasks. Some research indicates that initialis45 ing a network with transferable features from almost any number of layers can produce a boost to generalisation 
performance to a new dataset 27. It is desirable to have an image classification system that can use transferable ${ }_{105}$ features or utilise prior knowledge in image features in deep architectures for good generalisation in a wide range of applications. It is also imperative to understand how features in CNNs influence performance in general. For this objective we use a generative model to systematically generate, manipulate or disturb, and combine image ${ }^{110}$ features in terms of their scope and distribution and to study and compare the subsequent performances. The approach can also benefit the emerging zero-shot and oneshot learning paradigms [28, 29.

The Markov random field (MRF), associated with $_{115}$ visual perception of texture, is such a generative model and naturally provides a probabilistic way of encoding spatial contextual constraints that are essential in visual interpretation and image understanding [30, 31, 32, 33]. By characterising mutual influences using conditional probabilities, MRFs encode contextual dependencies among image pixels 34. Specifically, a clique-based structure makes MRFs particularly well-suited for capturing local relations and neighbourhood dependencies. In this paper, MRFs are used to study the properties of the features

70 learned by CNNs. They are also used to generate primitive image features directly or further combined with unsupervised learning for $\mathrm{CNNs}$ in order to investigate their performance variances.

Unsupervised learning of image features has recently 75 attracted a great deal of attention due to its advantages of separating feature learning and classification as well as not relying on huge amount of labelled training ${ }^{130}$ data. Principal component analysis network (PCANet) [23], discrete cosine transform network (DCTNet) and 80 wavelet scattering network (ScatNet) 22 are several well-known representatives, employing PCA, DCT and a wavelet scattering mechanism, respectively. The self- ${ }^{135}$ organising map (SOM) 35, a topological quantiser of data space, can provide an alternative to modelling contextual

85 constraints in image primitives and features. In this paper, several unsupervised methods of learning image properties (with PCA and SOM) are explored, together ${ }^{140}$ with generative features (DCT, Gabor and MRF). As a hybrid feature leaning mechanism, combining MRF with

90 SOM is proposed as an effective way of extracting dataindependent features in unsupervised fashion. By means of modelling different MRFs, a variety of spatial patterns are generated. A convolutional and translation-invariant SOM is employed to cluster these patterns to form compact filter

95 banks. Certain theoretical aspects of the proposed method are also discussed. We examine the effectiveness of the filter banks for image classification by using them in the first convolutional layer of CNNs. Various combinations of such MRF-SOM features are evaluated and compared with the same network architectures and hyper-parameter settings. The effectiveness of the proposed method has been verified on the MNIST [36, rotated MNIST [37, CIFAR-10 38] and CIFAR-100 38] image datasets.
The main contributions of the current work are summarised as follows:

1) A novel use of generative MRF models and SOM clustering for deriving efficient, data-independent features for $\mathrm{CNNs}$;

2) A novel use of generative features, in particular, MRFs, for determining the influence of image features on the performance of CNNs;

3) Comprehensive comparisons of unsupervised feature learning methods for CNNs.

The remainder of this paper is organised as follows. Section 2 gives a brief review of related work. Section 3 introduces different feature learning methods (unsupervised learning and handcrafted). The proposed hybrid $\mathrm{MRF}_{\text {Rot5-SOM }} \mathrm{SI}_{\mathrm{TI}}$ method is described and discussed in Section 4. Section 5 presents experimental results, together with the investigations on influence of various aspects of the early features. Conclusions are in Section 6 .

\section{Related Work}

For image classification, building a robust image representation is considered as a crucial step. The aim of feature extraction is to derive a set of discriminant representations or features that are suitable and useful for a given classification task. Features are often derived or extracted from raw data so that they are robust to the classification tasks, by minimising intra-class variability and same time maximising inter-class variability. Feature extraction broadly falls into three categories: handcrafted or designed, supervised learned and unsupervised learned.

Handcrafted features are (often manually) designed based on certain prior knowledge and domain properties. Representative examples include local image descriptors, dimensionality reduction and clustering methods. Scaleinvariant feature transformation (SIFT) 39], histogram of oriented gradient (HOG) [40], local binary pattern (LBP) 41 and the recent binary gradient pattern (BGP) 42 are typical image descriptors or handcrafted features by histograms or gradient information, for overcoming certain variations like illumination and occlusions. Often a balance or trade-off is struck between accuracy and computational efficiency.

Unsupervised learning can be used directly to group or dimensionally reduce image primitives to form meaningful representatives or features. Principal component analysis (PCA) and discrete cosine transform (DCT) are popular dimensionality reduction approaches to finding salient image features with lower computational complexity and have been extensively used in image processing tasks such as compression.

With the advent of deep learning especially CNNs, feature learning has recently shifted to brutally learning directly from massive amount of data. While such methodology is effective and extracted representations 
such as AlexNet 43 and VGG 44 can be used as the feature sets or initial features for fine tuning on different image classification tasks, these features are not transparent and difficult to interpret 45 . There exists ${ }^{215}$ work to visualise convolutional features in CNNs, broadly confirming the gradual increasing complexity across layers (e.g. 46, 47]). However, there is lack of study on how the scope of image features and their complexity affect the classification performance and on how an efficient set of features can be learned or derived for a specific task. 220

Many variants have been proposed to combine handcrafted with learnable features or deep architectures and arguably the first instance is the ScatNet 22. ScatNet computes translation invariant image representations and cascades wavelet transform convolutions with nonlinear 225 modulus and average operators. Such prefixed filter banks are used in similar multistage architectures of CNNs and have demonstrated the state-of-the-art performance in handwritten digits and texture recognition 22. PCANet 23 and DCTNet 25 have also shown to work well in face recognition by processing input images with a layerwise convolution with PCA and DCT filters, followed by binarisation, block-wise histograming. Dong et al. [26] proposed a compact unsupervised networks (CUNet), in which feature maps are derived by the $K$-means on image patches. The two-layer CUNet structure with weighted pooling has achieved performances comparable with the ScatNet, PCANet and DCTNet. The unsupervised way earning can significantly reduce computational load and overcome the requirement of massive labelled training samples. However, most of these existing approaches rely on an empirical histograming process and are limited by the filter size, weakening their generalisation ability.

Recent research has also demonstrated that initialising a deep supervised network with either handcrafted or transferable features can stabilise and generalise image representations especially in early layers and achieve

195 the state-of-the-art performances [27]. Oyallon et al. 48 proposed a scattering transform based deep hybrid network that uses the scattering network as the fixed initialisation for the first layer, followed by modern deep architectures. They suggested that early layers did not messarily need to be learned and predefined features could provide enlightenment on deep networks, making ${ }^{230}$ them to be more interpretable. Sarwar et al. 49 utilised Gabor filters to replace certain weight kernels in the convolutional layers. In conjunction with regular trainable 205 weights, their proposed CNN configuration obtained a balanced system with less computation and training time ${ }^{235}$ in exchange for a small tolerable degradation in accuracy. Mehrkanoon and Suykens [50] introduced a hybrid deep framework combining neural network architectures and kernel based models. Random Fourier features were used to compute an explicit feature map, which is then used to build kernels in the deep networks.

\section{Background}

In this section, two key mechanisms employed in the proposed feature learning for $\mathrm{CNNs}$ are reviewed: unsupervised learning (e.g. PCA and SOM) and predefined feature generation (e.g. DCT, Gabor and MRF).

\subsection{Principal Component Analysis}

The PCA 51, 52 approximates a high-dimensional input data space in a low-dimensional subspace spanned by the eigenvectors of the data covariance matrix.

Given a set of input vectors $\boldsymbol{X}=\left[\boldsymbol{x}_{1}, \boldsymbol{x}_{2}, \cdots, \boldsymbol{x}_{m}\right]$, a set of eigenvectors $\boldsymbol{V}=\left[\boldsymbol{v}_{1}, \boldsymbol{v}_{2}, \cdots, \boldsymbol{v}_{\tau}\right]$ corresponding to the largest eigenvalues are extracted from the covariance matrix $\boldsymbol{C}=\sum_{i=1}^{m}\left(\boldsymbol{x}_{i}-\overline{\boldsymbol{x}}\right)\left(\boldsymbol{x}_{i}-\overline{\boldsymbol{x}}\right)^{T}$, where $\overline{\boldsymbol{x}}$ is the mean vector. To generate PCA filter banks, we collect all overlapping patches of certain size from the input images and then extract a set of orthogonal eigenvectors as filters.

\subsection{Self-Organising Map}

The SOM reduces or maps an input space or a set of input vectors to a smaller set of prototype vectors topologically, as the representative of the input space. The prototypes or weights of neurons, $\boldsymbol{W}=\left[\boldsymbol{w}_{1}, \boldsymbol{w}_{2}, \cdots, \boldsymbol{w}_{d^{2}}\right]$ are commonly arranged in a $2 \mathrm{D}$ grid. When inner product or convolution is used instead of the Euclidean distance, the best matching unit at time step $t, b m u(t)$, is found as,

$$
b m u(t)=\arg \max _{i \in \Omega}\left[\boldsymbol{w}_{i}(t) \cdot \boldsymbol{x}(t)\right],
$$

or

$$
b m u(t)=\arg \max _{i \in \Omega}\left\{\boldsymbol{w}_{i}(t) * \boldsymbol{x}(t)\right\},
$$

and the corresponding weight updating rule becomes,

$$
\boldsymbol{w}_{i}(t+1)=\frac{\boldsymbol{w}_{i}(t)+\gamma(t) \eta(b m u, i, t)\left[\boldsymbol{x}(t)-\boldsymbol{w}_{b m u}(t)\right]}{\left\|\boldsymbol{w}_{i}(t)+\gamma(t) \eta(b m u, i, t)\left[\boldsymbol{x}(t)-\boldsymbol{w}_{b m u}(t)\right]\right\|},
$$

where $\Omega$ is the entire set of neuron indices, $\gamma(0<\gamma(t)<$ 1 ) is a monotonically decreasing learning rate, and $\eta$ is a neighbourhood function, e.g.,

$$
\eta(b m u, i, t)=\exp \left(-\frac{\left\|\boldsymbol{r}_{b m u}-\boldsymbol{r}_{i}\right\|^{2}}{2 \varpi(t)^{2}}\right),
$$

with $\boldsymbol{r}_{i}$ being the location of neuron $i$ on the map and $\varpi$ the width of the neighbourhood, decreasing with time.

The convolutional SOM clusters all image patches in a topology preserving manner and resulting weights of the neurons are used as filters. Note that the weights of the neurons are of the same dimensions as the image patches.

\subsection{Discrete Cosine Transform}

The DCT [53] decorrelates blocks of images (image patches) so that the transformed coefficients can be encoded independently. DCT expresses these blocks in terms of a sum of $2 \mathrm{D}$ cosine functions (basis functions) oscillating at gradually increasing frequencies in both directions 
and has been extensively used in image processing, esp. compression.

The 2D DCT basis functions are given as,

$B(u, v)=\sum_{i=0}^{M-1} \sum_{j=0}^{N-1} \frac{\Lambda(i) \Lambda(j)}{\sqrt{M N}} \cos \frac{\pi(2 i+1) u}{2 M} \cos \frac{\pi(2 j+1) v}{2 N}$

where

$$
\Lambda(\kappa)=\left\{\begin{array}{ll}
1 / \sqrt{2} & \kappa=0 \\
0 & \text { Otherwise }
\end{array},\right.
$$

$M$ and $N$ are the row and column size of the $B(u, v)$, espectively, and $u$ and $v$ represent horizontal and vertical ${ }^{28}$ frequencies. These predefined basis functions are considered as DCT filter banks.

\subsection{Gabor Filters}

Gabor filter is a band-pass filter that can be pre-tuned ${ }^{285}$ to certain spatial frequency (scale) and orientation 54. It has been shown that Gabor filters are able to model the receptive fields of simple cells in the visual cortex of some mammalian brains 55 .

The real and imaginary components of the Gabor filter representing orthogonal directions can be expressed as

$$
\Re[\psi(x, y)]=\frac{\rho^{2}}{\pi \lambda \zeta} \exp \left[-\left(\frac{\rho^{2}}{\lambda^{2}} x^{\prime 2}+\frac{\rho^{2}}{\zeta^{2}} y^{\prime 2}\right)\right] \cos \left(2 \pi \rho x^{\prime}\right),
$$

$$
\Im[\psi(x, y)]=\frac{\rho^{2}}{\pi \lambda \zeta} \exp \left[-\left(\frac{\rho^{2}}{\lambda^{2}} x^{\prime 2}+\frac{\rho^{2}}{\zeta^{2}} y^{\prime 2}\right)\right] \sin \left(2 \pi \rho x^{\prime}\right),
$$

where $x^{\prime}=x \cos \varphi+y \sin \varphi$ and $y^{\prime}=-x \sin \varphi+y \cos \varphi$.

$\rho$ is centre frequency and $\varphi$ denotes the orientation of the filter, $\lambda$ and $\zeta$ refer to the bandwidth along the $x$ and $y$ axes, respectively, and $\frac{\lambda}{\zeta}$ denotes the aspect ratio of the Gaussian function.

A standard Gabor filter bank of 5 scales and 8 orientations with $\lambda=\zeta=\sqrt{2}$ ) can be derived by varying ${ }_{290}$ the parameters $\rho$ and $\varphi$. The Gabor response of an input image is described as the convolution of the image with each of the Gabor filters.

\subsection{Markov Random Field}

Given an undirected graph $\mathcal{G} \triangleq(\mathcal{S}, \mathcal{N})$, a random field $F=\left\{F_{1}, F_{2}, \cdots, F_{s}\right\}$ is a group of jointly distributed random variables defined on a grid $\mathcal{S}$ with respect to a neighbourhood system $\mathcal{N}, s \in \mathcal{S}$. Each random variable takes a value $f_{i}$ from the label set $\mathcal{L}$. The random field ${ }_{300}$ satisfied:

1) Positivity: Any realisation is possible.

$$
P(f)>0, \forall f \in \mathbb{F},
$$

where $f=\left\{f_{1}, f_{2}, \cdots, f_{s}\right\}$ is a possible configuration and $\mathbb{F}$ denotes the set of all possible configurations of the field $F$.
2) Markovianity: The conditional probability of any site given the others only depends upon the configuration of its neighbours.

$$
P\left(f_{i} \mid f_{\mathcal{S}-\{i\}}\right)=P\left(f_{i} \mid f_{j}, j \in \mathcal{N}_{i}\right)=P\left(f_{i} \mid \mathcal{N}_{i}\right)
$$

where $\mathcal{S}-\{i\}$ denotes all the sites excluding site $i$, $f_{\mathcal{S}-\{i\}}$ is the set of labels at the sites in $\mathcal{S}-\{i\}$.

3) Homogeneity: The conditional probability $P\left(f_{i} \mid \mathcal{N}_{i}\right)$ depends only on the configuration of neighbours and is translation invariant.

To overcome the difficulty of specifying an MRF from the conditional probabilities [56], the Hammersley-Clifford theorem [57, 58. demonstrates the equivalence between MRFs and Gibbs distributions with regard to the same graph, providing a mathematically means of defining MRFs.

Let $P(f)$ denote a Gibbs distribution on the set $\mathcal{S}$. Then the joint probability $P(f)$ takes the form

$$
P(f)=\frac{1}{Z} e^{-\frac{1}{T} U(f)},
$$

where

$$
U(f)=\sum_{c \in \mathcal{C}} V_{c}(f)
$$

is the energy function that sums clique potentials $V_{c}(f)$ over all possible cliques $\mathcal{C}$ and stays positive for all possible configurations. $T$ is a constant called the temperature, and $Z$ is a normalising constant defined by

$$
Z=\sum_{f \in \mathbb{F}} e^{-\frac{1}{T} U(f)}
$$

which is also called the partition function.

Whilst MRFs only specify the conditional dependencies, Gibbs distributions provide explicit probability function for each clique. Based on the Markov-Gibbs equivalence, a random field $F$ is an $\mathrm{MRF}$ on $\mathcal{S}$ with respect to the neighbourhood system $\mathcal{N}$ if and only if the corresponding $P(f)$ is a Gibbs distribution, which indicates that the joint probability of any MRF can be measured by a Gibbs distribution, and every Gibbs distribution defines an MRF.

In computer vision, the intensity level of a single point in an image is highly dependent on the intensity levels of neighbouring pixels unless the image is simply random noise [59. As a stochastic process, MRFs regard the intensity levels in an image as random variables and model the conditional probability distributions between pixels and their neighbours.

Auto-models and multi-level logistic models 34, 60 are typical MRF models for establishing neighbourhood functions and clique potentials. Auto-models only take the single-site and pair-site cliques into account. Multi-level logistic models describe a clique potential depending on the type and the local configuration of that clique. In the 
case that only single-site and pair-site cliques are nonzero, the potential functions are written as

$$
V_{1}\left(f_{i}\right)=\alpha_{\mathcal{I}} \quad \text { if } f_{i}=\mathcal{I} \in \mathcal{L},
$$

$$
V_{2}\left(f_{i}, f_{j}\right)= \begin{cases}\beta_{c} & \text { if sites on clique }\{i, j\}=c \in \mathcal{C}_{2} \\ -\beta_{c} & \text { have the same label }\end{cases}
$$

where $\alpha_{\mathcal{I}}$ is the potential for label value $\mathcal{I} \in \mathcal{L}$, and $\beta_{c}$ defines the potentials for type $c$ pair-site cliques $\mathcal{C}_{2}$. In a first-order neighbourhood system, there can be four different parameter $\beta_{c}$ for four types of pairwise cliques. An isotropic multi-level logistic model share the same potential functions defined by $\beta$ and usually depicts bloblike regions, while an anisotropic model tends to generate texture-like images 64, 60, 61.

Given these constraints, several algorithms have been proposed to simulate MRF patterns [59, 34, 62. Among these approaches, the simulated annealing [34 is a practical and efficient method for approximating the global minimum of a given function as global optimal solution can be achieved as long as the cooling process is sufficiently slow.

\section{Proposed Feature Learning Approach and Frame- work}

In this paper, we use MRF to determine the influence of feature filters on CNNs by means of using and generating features of various spatial statistical properties. Then, we propose a hybrid feature learning approach $\mathrm{MRF}_{\mathrm{Rot} 5^{-365}}$ $\mathrm{SOM}_{\mathrm{TI}}$ combining the MRF and SOM to effectively derive data-independent feature filters with low complexity and computation. We first generate various MRF patterns, each of which is further rotated four additional times, hence giving in total five patterns. We denote these ${ }^{370}$ MRF patterns as $\mathrm{MRF}_{\text {Rot5 }}$. Although these patterns directly act as feature filters, we use a convolutional translation-invariant SOM to further cluster these feature patterns to reduce redundancies. This SOM is termed as $\mathrm{SOM}_{\mathrm{TI}}$. The combination of $\mathrm{MRF}$ and SOM makes it possible to model not only the contextual constraints

${ }_{335}$ lying within each filter but also the topological structure between different filters. On one hand, the MRF provides flexible and natural models for the interaction between spatially related random variables in their neighbourhood systems 63. For each individual MRF pattern, there a specified probability distribution that reveals the conditional constraints between neighbouring pixels within that pattern. On the other hand, the SOM forms a discrete mapping of the feature space with certain topological relationships. The general block diagram of the proposed $\mathrm{MRF}_{\text {Rot5-SOM}} \mathrm{SOM}_{\mathrm{TI}}$ method is depicted in Fig. 1 .

The obtained $\mathrm{MRF}_{\text {Rot5-SOM}}-\mathrm{SO}_{\mathrm{TI}}$ filter bank can be used as the first layer of the CNNs. The resulting
$\mathrm{MRF}_{\text {Rot5-SOM }} \mathrm{SI}_{\mathrm{TI}} \mathrm{CNN}$ architecture explores an image classification framework by fusing the respective strengths of $\mathrm{MRF}_{\mathrm{Rot} 5}-\mathrm{SOM}_{\mathrm{TI}}$ and $\mathrm{CNNs}$ to bring the expressive power of deep architecture to probability formulations and unsupervised feature learning algorithms. Instead of learning convolutional layer in supervised mode, these pre-generated $\mathrm{MRF}_{\text {Rot5 }}-\mathrm{SOM}_{\mathrm{TI}}$ filter banks considerably reduce training time with comparable performances.

\subsection{Generating Various Markov Random Field Patterns}

As a statistical model, the main advantage of MRFs is that they model pixels dependency together with their spatial constraints and this in turn can be used for feature generation. We define multi-level logistic MRF models (label set $\mathcal{L}=\{0,1\}$ ) with parameters $\beta$ specified on a fifth-order neighbourhood system as,

$$
\begin{aligned}
\beta= & \left(\beta_{11}, \beta_{12} ; \beta_{21}, \beta_{22} ; \beta_{31}, \beta_{32} ; \beta_{41}, \beta_{42} ; \beta_{51}, \beta_{52}\right) \\
= & {\left[\begin{array}{ccccc}
\beta_{51} & \beta_{41} & \beta_{32} & \beta_{42} & \beta_{52} \\
\beta_{41} & \beta_{21} & \beta_{12} & \beta_{22} & \beta_{42} \\
\beta_{31} & \beta_{11} & 0 & \beta_{11} & \beta_{31} \\
\beta_{42} & \beta_{22} & \beta_{12} & \beta_{21} & \beta_{41} \\
\beta_{52} & \beta_{42} & \beta_{32} & \beta_{41} & \beta_{51}
\end{array}\right] . }
\end{aligned}
$$

By varying the values of $\beta$, various spatial distribution properties can be generated using the simulated annealing strategy. For example, large $\beta_{11}$ promotes short horizontal line segments, while $\beta_{52}$ specifies the correlation among long diagonal pixels. The detailed algorithm is described in Algorithm 11. To further improve the pattern diversity with respect to rotation, each generated MRF pattern is rotated uniformly to four different angles within 45 degrees. Fig. 2(a) shows 16 samples taken from specified multi-level logistic distributions displaying horizontal, vertical and diagonal line textures, whilst Fig. 2(b) displays 16 natural patterns covering a broad range, from checkerboard-like patterns to blob-like textures, generated with randomly distributed $\beta$ values.

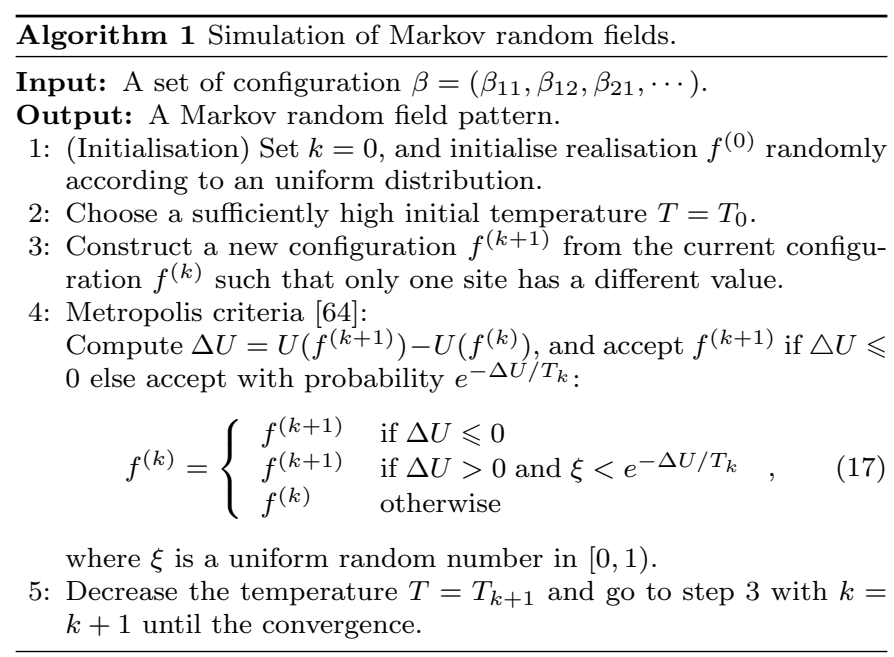

With sample images generated, we extract filters by randomly selecting $p \times p$ patches from each image and 


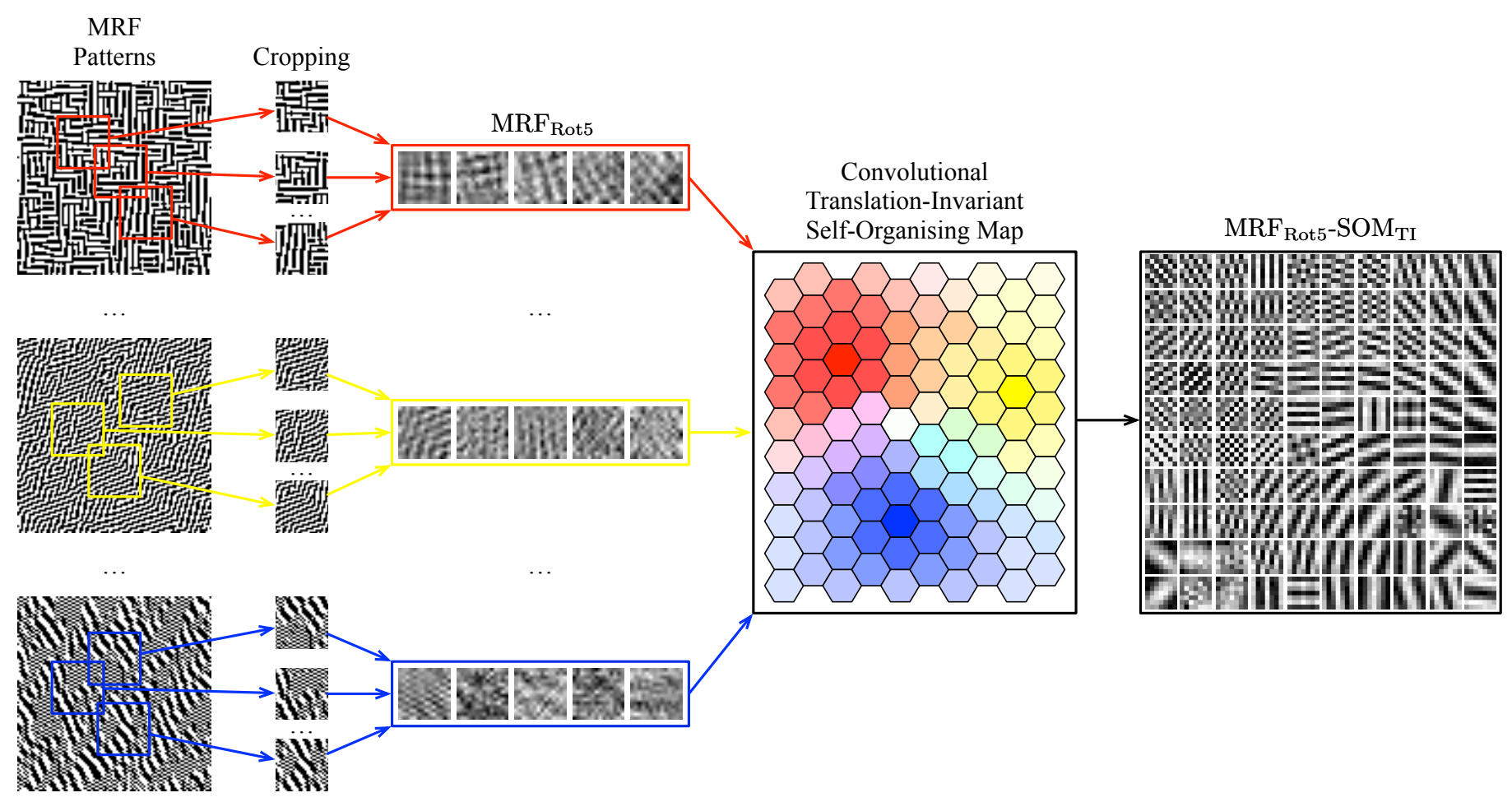

Figure 1: Block diagram of proposed $\mathrm{MRF}_{\mathrm{Rot} 5}-\mathrm{SOM}_{\mathrm{TI}}$ method.

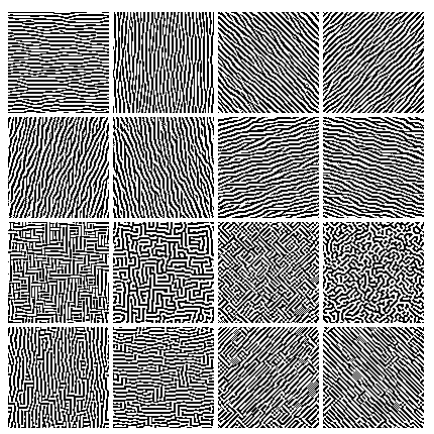

(a)

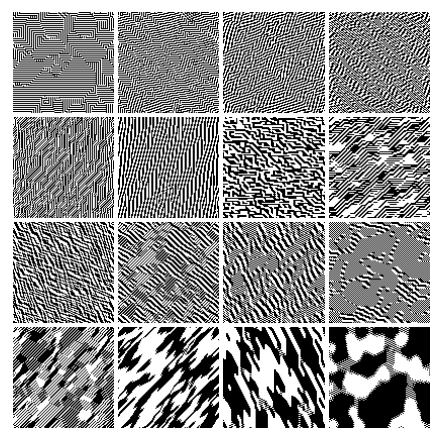

(b)

Figure 2: Sample images generated from the multi-level logistic MRF models for (a) specified and (b) random parameters.

repeat the process multiple times. The resulting patches are the average of those patches from each sample pattern.

Fig. 3 shows examples of $14 \times 14$ patches extracted from the samples in Figs. 2(a) and 2(b) respectively.

\subsection{Filter Clustering Using Convolutional Translation- Invariant Self-Organising Map}

Generating MRF patterns using randomly distributed parameters offers patterns with a wide range of spatial properties. However, some of the resulting patches are duplicative thus leading to redundant filters with similar features. Furthermore, despite the use of averaging, there still exists redundancy or unwanted noise in the representative patches, which may affect the subsequent filtering and classification processes. Therefore, to remove

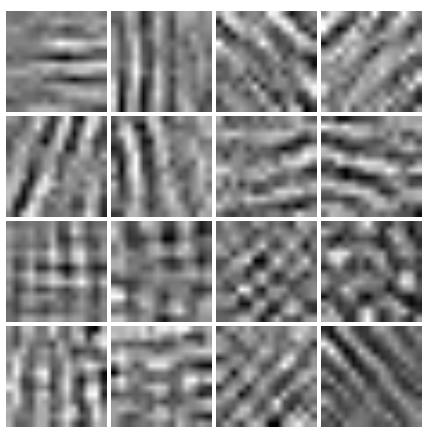

(a)

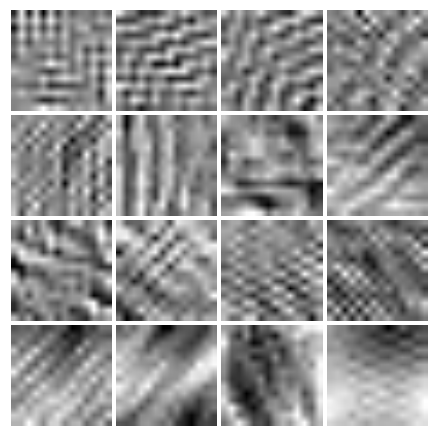

(b)
Figure 3: Sampled $14 \times 14$ patches, (a) from MRF patterns in Fig. 2(a) and (b) from MRF patterns in Fig. 2(b)

redundancies and reduce noise, we propose to use a translation-invariant training scheme of SOM to cluster these feature patches.

Let us denote the feature patches derived from the MRF patterns by

$$
\Theta=\left[\boldsymbol{\theta}_{1}, \boldsymbol{\theta}_{2}, \cdots, \boldsymbol{\theta}_{n}\right] \in \mathbb{R}^{p^{2} \times n} .
$$

For each patch $\boldsymbol{\theta}_{h}$ indexed by $h$, we collect all overlapping filter blocks using a $q \times q$ sliding window with step size 1 , which produces a total number of $(p-q+1)^{2}$ blocks as

$$
\Xi=\left[\boldsymbol{\vartheta}_{1}^{(h)}, \boldsymbol{\vartheta}_{2}^{(h)}, \cdots, \boldsymbol{\vartheta}_{(p-q+1)^{2}}^{(h)}\right] \in \mathbb{R}^{q^{2} \times(p-q+1)^{2}} .
$$

At each time step $t$, the distances between each block $\boldsymbol{\vartheta}_{l}^{(h)}$ and each neuron weight $\boldsymbol{w}_{i}$ in the SOM are calculated 
using the convolutional metric. The resulting distance matrix has the size of $(p-q+1)^{2} \times d^{2}$, where $d^{2}$ denotes the ${ }_{415}$ number of neurons in the map. A winner-take-all scheme is employed to find the best matching unit as the winning neuron of the winning block,

$$
\begin{gathered}
\operatorname{bmu}(t)=\arg \min _{l, i} \operatorname{dist}\left\langle\boldsymbol{\vartheta}_{l}^{(h)}, \boldsymbol{w}_{i}\right\rangle=\arg \max _{l, i}\left\{\boldsymbol{\vartheta}_{l}^{(h)} * \boldsymbol{w}_{i}\right\}, \quad{ }_{420} \\
l=1,2, \cdots,(p-q+1)^{2} . i=1,2, \cdots, d^{2} .
\end{gathered}
$$

where * denotes 2D convolution. The best matching unit and its associated neighbouring neurons are updated according to the winning block and neighbourhood function. During the training, the effective range of the neighbourhood and learning rate monotonically decrease with time until the convergence.

The detailed training procedure for the convolutional, translation-invariant SOM is described in Algorithm 2, The resulting clustered filter blocks are considered as the extracted feature filters to be used as prefixed filter banks in the early convolutional layers of the CNN for image classification.

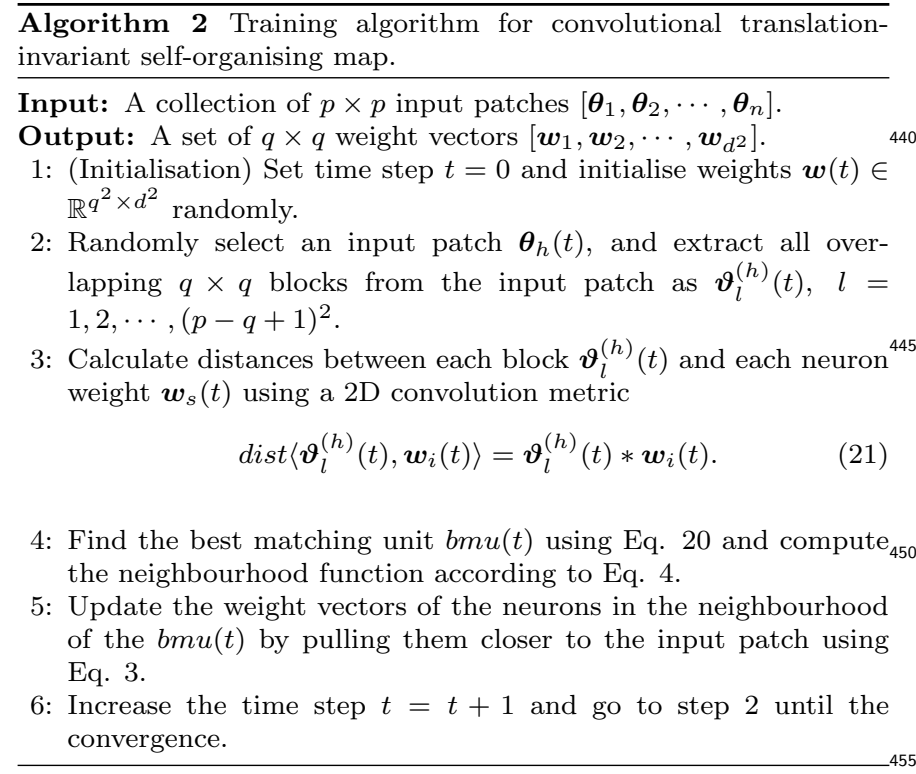

\subsection{Convergence Properties}

The proposed method consists of two main algorithms: the synthesis of MRFs (Algorithm 1) and the convolutional translation-invariant SOM (Algorithm 2). These two algorithms have solid theoretical backgrounds for reproducibility and convergence, and can guarantee the statistical properties of the generated features and learned prototypes (in unsupervised way).

The generartion of MRFs is mainly based on the Metropolis criteria 64] and simulated annealing strategy 465 34. According to Hammersley 65, the convergence of the Metropolis method is guaranteed, and the application of the algorithm will eventually result in a set of configurations with desired distribution 59. The simulated annealing approximates the global minimum of a given function, and the convergence has been proved with a prescribed schedule for lowering the temperature $T$. At a lower temperature, lower energy configurations are more probable and the couplings between neighbouring pixels are tighter. Higher temperature induces looser couplings and more chaotic appearance to the configurations as the energy $U(f)$ is less important in determining its likelihood $P(f)$ (Eqs. 10, 11, 12). The annealing process gradually lowers the temperature and drives the configurations to their low energy, highly regular and stable states. Experimental results indicate that a sufficiently high initial temperature and a gradually decreasing annealing schedule produce low energy states or the most probable states under the Gibbs distribution.

As a variant of SOM, the training algorithm for proposed convolutional translation invariant SOM shares similar convergence properties as the traditional SOM. Kohonen [66] and Cottrell and Fort [67] have proved that a one-dimensional SOM with a one-step neighbourhood function will converge to a stable ordered configuration as a map of the input space. Erwin et al. 68 further extended the proof to any monotonically decreasing neighbouring function and a constant learning step size. Yin and Allinson [69] and Yin [70] have shown that under some mild assumptions of the learning rate the neurons tends to multiple Gaussian distributed stochastic processes, eventually converging to the probability distribution of training data. In the case that weight vectors are normalised (Eq. 3), the Euclidean distance and the dot product measure are equivalent: $\left\|\boldsymbol{w}_{i}-\boldsymbol{x}\right\|^{2}=\left\|\boldsymbol{w}_{i}\right\|^{2}+\|\boldsymbol{x}\|^{2}-2 \boldsymbol{w}_{i} \cdot \boldsymbol{x}$. Thus $\min \left(\left\|\boldsymbol{w}_{i}-\boldsymbol{x}\right\|^{2}\right)=\max \left(\boldsymbol{w}_{i} \cdot \boldsymbol{x}\right)$ when $\left\|\boldsymbol{w}_{i}\right\|^{2}$ is normalised as it is. This can also be verified visually by observing th similarity of the domination regions for these two distance measures 71. The convergence properties is extendable to the convolutional translation-invariant SOM since the convolution measure coincides with the dot product as $\boldsymbol{w}_{i} * \boldsymbol{x}=\operatorname{rot}_{180}\left(\boldsymbol{w}_{i}\right) \cdot \boldsymbol{x}$. Practically, as only limited data samples are used and training is performed in finite time, the number of iterations has to be reasonably large to ensure a reasonable organisation of the map. However, the advantage of using such learning mechanism is that these filters can be learned separately from the remaining $\mathrm{CNN}$, which is trained in the usual supervised fashion.

\section{Experiments and Discussion}

This section presents the experiments that were conducted to evaluate the proposed feature generation process, influence of the feature filters in CNNs, and the proposed $\mathrm{MRF}_{\text {Rot5 }}-\mathrm{SOM}_{\mathrm{TI}}-\mathrm{CNN}$ framework. The four benchmark datasets used are first described in 5.1 Subsection 5.2 explains the feature generation process, along with a list of all symbols. Subsections 5.3 and 5.4 
present the experimental results on the four datasets and comparisons with other state-of-the-art methods.

\subsection{Datasets}

\subsubsection{MNIST Handwritten Digits dataset}

The MNIST dataset [36] of handwritten digits, formed from the larger NIST dataset, is a commonly used image ${ }^{525}$ dataset in image processing and machine learning. The

475 dataset contains 60000 training images and 10000 test images, normalised and centred to the size of $28 \times 28$ pixels.

\subsubsection{Rotated MNIST dataset}

530

The rotated MNIST dataset 37 was generated from the original MNIST to introduce more controlled varia-

480 tions (rotation angles). Digits were rotated by an angle generated uniformly between 0 and $2 \pi$ radians.

\subsubsection{CIFAR-10 Image dataset}

The CIFAR-10 image dataset 38 is a collection of $6000032 \times 32$ colour images split in 10 classes (airplane, automobile, bird, cat, deer, dog, frog, horse, ship and truck) with 6000 images per class. The training set consists of 50000 training images and 10000 testing images, both containing equal numbers of samples for each class. Whilst each image contains one notable instance of its respective class object, there are also variations in viewpoint and scale.

\subsubsection{CIFAR-100 Image dataset}

Similar to the CIFAR-10, the CIFAR-100 image dataset 38] consists of $6000032 \times 32$ colour images. There are 20 superclasses in total split into 100 classes with 500 training and 100 testing images per class. Each image is labelled with a "fine" label (the class to which it belongs) and a "coarse" label (the superclass to which it belongs).

\subsection{Feature Generation and CNN Training}

To generate a wide range of MRF patterns, we varied the values of parameter $\beta$ to model different distributions using the multi-level logistic MRF models with a fifth-order neighbourhood system. The patterns ${ }_{560}$ were initialised randomly with a size of $100 \times 100$ from iform distribution, and then were simulated using 500 iterations of a Metropolis sampler with the simulated annealing strategy (Algorithm 1). The temperature was initialised at $T=1$ and annealed by a factor of ${ }_{565}$ 0.999 per iteration. Consequently, over four thousands MRF patterns were simulated. Together with four rotations, there were over twenty thousand resultant MRF patterns. Within each MRF sample pattern, we randomly selected $5000 p \times p$ patches at different locations and averaged them as the representative patch for the sample pattern.

In the filter clustering and reduction step, the convolutional translation invariant SOM was trained on these patches to produce a total number of $d \times d$ filters, each of size of $q \times q$ (Algorithm 2). In each $p \times p$ patch, we collected a total number of $(p-q+1)^{2}$ overlapping $q \times q$ blocks. Under the circumstance that filters were applied to RGB images with three channels, we repeated the aforementioned patch selection process three times into three separate channels. This leads to the three patches representing the same MRF pattern but with small variations. The remaining steps stayed unchanged resulting in input patches and filters of sizes, $p \times p \times 3$ and $q \times q \times 3$, respectively. The SOM was then trained to derive the most representative filters invariant to translation, small rotation and distortion. The filter width (or height) $q$ was set to the half of patch width (or height) $p$. Gaussian function and convolution metric were chosen as the neighbourhood function and similarity measure, respectively. During the training, the learning rate $\gamma$ starting at 0.1 was linearly decreased to 0.05 at time step $t=1000000$. The effective range of the neighbourhood $\varpi$ initialised as the half of the width (or height) of the map $d$ was annealed at the same speed as the learning rate. The total number of iterations was fixed at 5000000. The converged or trained weights were considered as the filter banks in the adopted CNNs.

To validate the proposed feature learning mechanism, we further compared the $\mathrm{MRF}_{\text {Rot5}}-\mathrm{SOM}_{\mathrm{TI}}$ features with four variants, MRF-SOM, MRF-SOM $\mathrm{MRF}_{\text {Rot5}}$-SOM. MRF-SOM and MRF-SOM $\mathrm{TI}_{\mathrm{T}}$ refer to randomly generated raw MRF patterns clustered by traditional convolutional SOM and the proposed translationinvariant convolutional SOM, respectively. MRF $\mathrm{Mr}_{\text {Rot }}$ denotes the raw MRF features with four rotated offspring sets, while $\mathrm{MRF}_{\text {Rot5}}-\mathrm{SOM}$ and $\mathrm{MRF}_{\text {Rot5 }}-\mathrm{SOM}_{\mathrm{TI}}$ were derived by applying two different SOM training processes on $\mathrm{MRF}_{\text {Rot5 }}$ patterns.

During the training of CNN for image classification, we initialised the convolutional weights in the model from a normal distribution with zero biases. $\mathrm{MRF}_{\mathrm{Rot} 5}-\mathrm{SOM}_{\mathrm{TI}}$ trained weights were considered as the prefixed filter bank for the first convolutional layer of CNN and remained the same. Zero padding with a size of $(q-1) / 2$ was applied to make sure that the output dimensions were the same as the inputs. The model was trained for 300 epochs using stochastic gradient descent with a batch size of 200 . Weight decay and momentum were set to 0.0001 and 0.9 , respectively. The learning rate initialised with 0.01 was annealed by a factor of 0.989 per epoch.

For clarity, all the symbols or parameters used in the paper and their meanings are listed in Table 1.

\subsection{Experiments on MNIST and Rotated MNIST Datasets}

The MNIST was used to investigate the influence of feature distribution, filter size, number of filters and types of filters in the proposed $\mathrm{MRF}_{\mathrm{Rot} 5}-\mathrm{SOM}_{\mathrm{TI}}-\mathrm{CNN}$. We then compared the performance of $\mathrm{MRF}_{\text {Rot5- }}-\mathrm{SOM}_{\mathrm{TI}}-\mathrm{CNN}$ against that of the state-of-the-art models on both MNIST and rotated MNIST datasets. 
Table 1: List of symbols.

\begin{tabular}{|c|c|}
\hline Symbols & Meaning \\
\hline $\boldsymbol{X}=\left[\boldsymbol{x}_{1}, \boldsymbol{x}_{2}, \cdots, \boldsymbol{x}_{m}\right]$ & Input vectors \\
\hline $\boldsymbol{V}=\left[\boldsymbol{v}_{1}, \boldsymbol{v}_{2}, \cdots, \boldsymbol{v}_{\tau}\right]$ & PCA eigenvectors \\
\hline$\overline{\boldsymbol{x}}$ & Mean of the inputs \\
\hline$\tau$ & Number of eigenvectors \\
\hline$m$ & Number of input vectors \\
\hline $\boldsymbol{W}=\left[\boldsymbol{w}_{1}, \boldsymbol{w}_{2}, \cdots, \boldsymbol{w}_{d^{2}}\right]$ & SOM neuron weights \\
\hline 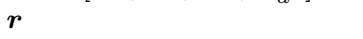 & Locations of neurons on the map \\
\hline bmu & Best matching unit \\
\hline$\gamma$ & SOM learning rate \\
\hline$\eta$ & SOM neighbourhood function \\
\hline$\varpi$ & $\begin{array}{l}\text { The effective range of the } \\
\text { neighbourhood }\end{array}$ \\
\hline$B(u, v)$ & 2D DCT bases \\
\hline$\Lambda$ & DCT coefficients \\
\hline$M$ & Number of rows in $B(u, v)$ \\
\hline$N$ & Number of columns in $B(u, v)$ \\
\hline$\psi(x, y)$ & $\begin{array}{l}\text { Impulse response of } 2 \mathrm{D} \text { Gabor } \\
\text { filter }\end{array}$ \\
\hline$\lambda$ & Bandwidth along the $x$ axis \\
\hline$\zeta$ & Bandwidth along the $y$ axis \\
\hline$\rho$ & Centre frequency \\
\hline$\varphi$ & Orientation of the filter \\
\hline$x$ & Location along the $x$ axis \\
\hline$y$ & Location along the $y$ axis \\
\hline $\mathcal{G}$ & Undirected graph \\
\hline $\mathcal{N}$ & Neighbourhood system \\
\hline $\mathcal{S}$ & Sites in the graph \\
\hline $\mathcal{L}$ & Label set \\
\hline$F=\left\{F_{1}, F_{2}, \cdots, F_{s}\right\}$ & A random field \\
\hline$f=\left\{f_{1}, f_{2}, \cdots, f_{s}\right\}$ & $\begin{array}{l}\text { One configuration of the random } \\
\text { field } F\end{array}$ \\
\hline $\mathbb{F}$ & $\begin{array}{l}\text { All possible configurations of the } \\
\text { random field } F\end{array}$ \\
\hline$U$ & Energy function \\
\hline$V_{c}$ & Clique potentials \\
\hline $\mathcal{C}$ & All possible cliques \\
\hline$Z$ & Partition function \\
\hline$s$ & Number of random variables \\
\hline$\alpha, \beta$ & MRF model parameters \\
\hline$\xi$ & $\begin{array}{l}\text { An uniformly distributed random } \\
\text { number in }[0,1)\end{array}$ \\
\hline $\boldsymbol{\Theta}=\left[\boldsymbol{\theta}_{1}, \boldsymbol{\theta}_{2}, \cdots, \boldsymbol{\theta}_{n}\right]$ & Input patches \\
\hline$\Xi=$ & Overlapping filter blocks in \\
\hline$\left[\boldsymbol{\vartheta}_{1}^{(h)}, \boldsymbol{\vartheta}_{2}^{(h)}, \cdots, \boldsymbol{\vartheta}_{(p-q+1)^{2}}^{(h)}\right]$ & $h^{t h}$ patch $\boldsymbol{\theta}_{h}$ \\
\hline$p$ & Width or height of input patches \\
\hline$q$ & Width or height of filters \\
\hline$n$ & Number of input patches \\
\hline$d^{2}$ & Number of neurons in the SOM \\
\hline$\mu$ & Mean of Normal distribution \\
\hline$\sigma$ & $\begin{array}{l}\text { Standard deviation of Normal } \\
\text { distribution }\end{array}$ \\
\hline$h, i, j, k, u, v, \kappa$ & Indices \\
\hline$t$ & Time step \\
\hline$T$ & Temperature \\
\hline
\end{tabular}

\subsubsection{Data Pre-Processing and Network Architecture}

For MNIST and rotated MNIST datasets, the only pre- ${ }_{615}$ processing taken was mean subtraction.

The architecture consists of 9 major layers excluding the input as illustrated in Table 2. The first convolutional layer uses a prefixed filter bank with zero padding and the filter parameters remain the unchanged during ${ }_{620}$ training, followed by a $2 \times 2$ max-pooling layer, the second convolutional layer has filters of size $1 \times 1$, each of which learns a linear combination of the prefixed filter bank and compresses the feature maps. Following another convolution-pooling block, a dropout layer is used to prevent overfitting. Finally, two fully connected layers are used with a softmax layer to produce probabilistic outputs over classes. In this architecture, batch normalisation is inserted in each convolutional layer between the convolution and activation, excluding the first and last layers. Besides, we also trained a baseline model with the same network architecture as a benchmark, with the training strategy and hyperparameters kept the same except that weights of the first convolutional layer were learned by the standard stochastic gradient descent.

Table 2: Architecture used on MNIST and rotated MNIST datasets.

\begin{tabular}{c}
\hline Input $28 \times 28$ Grayscale Images \\
\hline$q \times q$ conv. $d^{2}$ ReLU with zero padding $(q-1) / 2$ \\
$1 \times 1$ conv. 50 ReLU \\
$2 \times 2$ max-pooling \\
\hline Dropout prob $=0.5$ \\
\hline $3 \times 3$ conv. 50 ReLU \\
$3 \times 3$ conv. 50 ReLU \\
$2 \times 2$ max-pooling \\
\hline Dropout, prob $=0.5$ \\
\hline $3 \times 3$ conv. 200 ReLU \\
$3 \times 3$ conv. 200 ReLU \\
\hline Dropout, prob $=0.5$ \\
$1 \times 1$ conv. 50 ReLU \\
$1 \times 1$ conv. 10 ReLU \\
\hline 10 -class softmax
\end{tabular}

\subsubsection{Impact of Feature Distribution}

Firstly, we investigated how the spatial distributions of the feature filters impact on the performance of CNNs. Most current work in the literature only visualises the learned features in CNNs. However, little has been done to probe how well the feature distribution should be. Such a study is of particular importance to in-depth understanding of feature learning and feature generation, underlined by this paper. MRFs provide a natural mechanism to study feature distributions in a generative way. For this purpose, feature filters generated according to various spatial distributions of the MRF parameters (from Algorithms 1 and 2) are used in the CNNs for image classification. Specifically, we generated a set of feature filters from the MRF parameters that were all normally distributed with $(\mu=0, \sigma=1)$ against several skewed feature sets from the MRF parameters that were shifted from normal distributions. These MRF patterns were then clustered using the convolutional invariant SOMs, producing several differently distributed $40 \times 40$ feature maps with filter size $7 \times 7$. Results for these cases, presented in Table 3, show that the broader the spatial properties of the feature filters the better the performance. This result is intuitive, indicating that the basic features should be well distributed, and clearly MRFs provide a perfect means for this purpose. 
Table 3: Error rate (\%) for different properties of MRF filters on MNIST dataset.

\begin{tabular}{|c|c|c|c|c|}
\hline \multirow[b]{2}{*}{$\begin{array}{l}\text { MRF } \\
\text { Order }\end{array}$} & \multicolumn{2}{|c|}{ MRF Parameters } & \multirow[b]{2}{*}{ Orientation } & \multirow[b]{2}{*}{$\begin{array}{c}\text { Error } \\
\text { Rate }(\%)\end{array}$} \\
\hline & $\begin{array}{l}\text { Normal } \\
(\mu=0 \\
\sigma=1)\end{array}$ & $\begin{array}{l}\text { Skewed } \\
(\mu=4 \\
\sigma=1)\end{array}$ & & \\
\hline \multirow{5}{*}{5} & $\begin{array}{l}\beta_{12}, \beta_{21}, \beta_{22}, \beta_{32} \\
\beta_{41}, \beta_{42}, \beta_{51}, \beta_{52}\end{array}$ & $\beta_{11}, \beta_{31}$ & Horizontal & $0.32 \pm 0.01$ \\
\hline & $\begin{array}{l}\beta_{11}, \beta_{21}, \beta_{22}, \beta_{31} \\
\beta_{41}, \beta_{42}, \beta_{51}, \beta_{52}\end{array}$ & $\beta_{12}, \beta_{32}$ & Vertical & $0.36 \pm 0.02$ \\
\hline & $\begin{array}{c}\beta_{11}, \beta_{12}, \beta_{22}, \beta_{31} \\
\beta_{32}, \beta_{42}, \beta_{52}\end{array}$ & $\begin{array}{c}\beta_{21}, \beta_{41} \\
\beta_{51}\end{array}$ & $\begin{array}{c}\text { Main } \\
\text { Diagonal }\end{array}$ & $0.40 \pm 0.03$ \\
\hline & $\begin{array}{c}\beta_{11}, \beta_{12}, \beta_{21}, \beta_{31} \\
\beta_{32}, \beta_{41}, \beta_{51}\end{array}$ & $\begin{array}{c}\beta_{22}, \beta_{42} \\
\beta_{52}\end{array}$ & $\begin{array}{l}\text { Counter } \\
\text { Diagonal }\end{array}$ & $0.39 \pm 0.02$ \\
\hline & $\begin{array}{c}\beta_{11}, \beta_{12}, \beta_{21}, \beta_{22} \\
\beta_{31}, \beta_{32}, \beta_{41}, \beta_{42} \\
\beta_{51}, \beta_{52}\end{array}$ & - & All & $\mathbf{0 . 3 0} \pm 0.01$ \\
\hline 2 & $\beta_{11}, \beta_{12}, \beta_{21}, \beta_{22}$ & - & All & $\mathbf{0 . 3 0} \pm 0.02$ \\
\hline
\end{tabular}

\subsubsection{Impact of Filter Size}

Then, we investigated the choice of filter size (local receptive field) in the proposed $\mathrm{MRF}_{\mathrm{Rot} 5}-\mathrm{SOM}_{\mathrm{TI}}-\mathrm{CNN}$

framework. For this purpose, we varied the filter size from $3 \times 3$ to $9 \times 9$ with the size of zero padding from $1 \times 1$ to $4 \times 4$, while the total number of filters (size of the SOM) in the first convolutional layer was fixed to $40 \times 40$. The same network architecture and training parameters were used. Table 4 lists the results of the network performances against varying sizes of filters on various datasets. Clearly, for the MNIST and rotated MNIST datasets, the $7 \times 7$ filters achieved the minimum error rate, that is, moderate local receptive fields are better than either small or large ones. Results on other datasets reveal that filter size can influence the performance to certain extent and the optimal filter size depends on data and can be selected empirically as often is. It is interesting to note that small sizes (esp. $3 \times 3$ ) appear to generalise well across all datasets, supporting the practice used in many well-known structures such as in VGG, GoogleNet and ResNet.

Table 4: Error rate (\%) for various filter sizes on MNIST, rotated MNIST, CIFAR-10 and CIFAR-100 datasets.

\begin{tabular}{ccc}
\hline Dataset & Filter Size & Error Rate (\%) \\
\hline \multirow{3}{*}{ MNIST } & $3 \times 3$ & $0.32 \pm 0.02$ \\
& $5 \times 5$ & $0.32 \pm 0.02$ \\
& $7 \times 7$ & $\mathbf{0 . 3 0} \pm 0.01$ \\
& $9 \times 9$ & $0.35 \pm 0.04$ \\
\hline \multirow{3}{*}{ Rotated MNIST } & $3 \times 3$ & $2.84 \pm 0.04$ \\
& $5 \times 5$ & $2.70 \pm 0.04$ \\
& $7 \times 7$ & $\mathbf{2 . 6 9} \pm 0.02$ \\
CIFAR-10 & $9 \times 9$ & $2.83 \pm 0.05$ \\
\hline & $3 \times 3$ & $\mathbf{8 . 3 0} \pm 0.13$ \\
& $5 \times 5$ & $8.88 \pm 0.11$ \\
& $9 \times 7$ & $9.61 \pm 0.10$ \\
CIFAR-100 & $9 \times 9$ & $10.25 \pm 0.12$ \\
& $3 \times 3$ & $\mathbf{3 3 . 2 5} \pm 0.15$ \\
& $5 \times 5$ & $33.72 \pm 0.21$ \\
& $7 \times 7$ & $34.79 \pm 0.23$ \\
& $9 \times 9$ & $36.70 \pm 0.42$ \\
\hline
\end{tabular}

\subsubsection{Impact of Number of Filters}

We varied the number of filters in the first layer of CNN (SOM size) from $10 \times 10$ to $50 \times 50$ with fixed filter size of $7 \times 7$. The experiments were repeated several times for good precision. Results presented in Table 5 show that by increasing the number of filters, the classification accuracy improved, though the rate of improvement significantly slowed after a certain number. However, a larger number of filters would incur larger parameter space and longer training time. This value could be a trade-off between performance and computational complexity.

Table 5: Error rate (\%) for different number of filters on MNIST dataset.

\begin{tabular}{ccc}
\hline SOM Size & \#Filters & Error Rate $(\%)$ \\
\hline $10 \times 10$ & 100 & $0.32 \pm 0.02$ \\
$20 \times 20$ & 400 & $0.32 \pm 0.02$ \\
$30 \times 30$ & 900 & $0.30 \pm 0.03$ \\
$40 \times 40$ & 1600 & $0.30 \pm 0.01$ \\
$50 \times 50$ & 2500 & $0.30 \pm 0.01$ \\
\hline
\end{tabular}

\subsubsection{Comparisons of Different Types of Filters}

The effect of different types of filters was also studied and compared with the proposed feature learning method, $\mathrm{MRF}_{\text {Rot5 }}-\mathrm{SOM}_{\mathrm{TI}}$. Similar to the previous experiment, we fixed the filter size to $7 \times 7$ and compared the proposed $\mathrm{MRF}_{\text {Rot5 }}-\mathrm{SOM}_{\mathrm{TI}}$ filters with PCA components, DCT bases, Gabor filters, MRF patterns, SOM weights and some combinations. PCA filters were learned following the same protocol as the PCANet 23. For both PCA filters and DCT bases, the maximum number of filters obtainable are limited by the filter size. For Gabor filters, we derived 40 multi-scale and multi-orientations ( 5 scales and 8 orientations) and for MRF patterns, 100 were randomly selected. SOM weights were trained from collected 9 overlapping patches with a size of $14 \times 14$ and step size of 7 from each MNIST image. Then the convolutional translation-invariant SOM was trained to obtain the filter bank containing $1007 \times 7$ filters. Fig. 4 displays the resulting nine different filter banks.

Table 6 lists the performances of the network with these prefixed filter banks on the MNIST dataset. The results show that among these methods, the proposed $\mathrm{MRF}_{\text {Rot5- }}$ $\mathrm{SOM}_{\mathrm{TI}}$ achieved the best performance with comparable number of filters.

\subsubsection{Comparisons with the State-of-the-Art Methods}

We trained $\mathrm{CNN}$ with $\mathrm{MRF}_{\text {Rot5}}-\mathrm{SOM}_{\mathrm{TI}}$ based filter banks and repeated the trial multiple times to obtain the mean error rate and standard deviation. For the MNIST dataset, among the 10000 test images, only 30 samples on average were wrongly recognised. Fig. 5 displays all the misclassified samples of one typical run and the predicted probabilities associated with each class box. It can be seen that most of these wrongly classified 


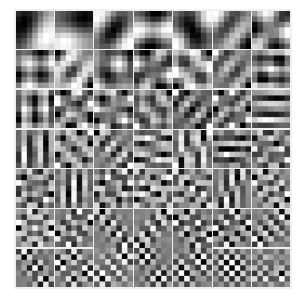

(a)

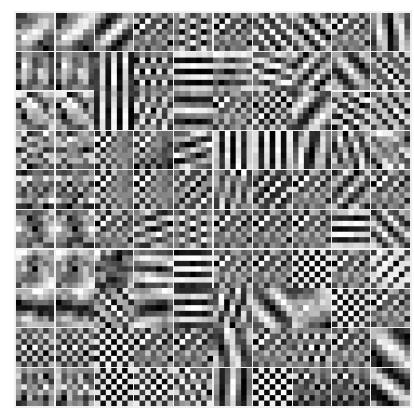

(f)

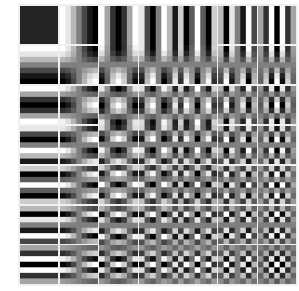

(b)

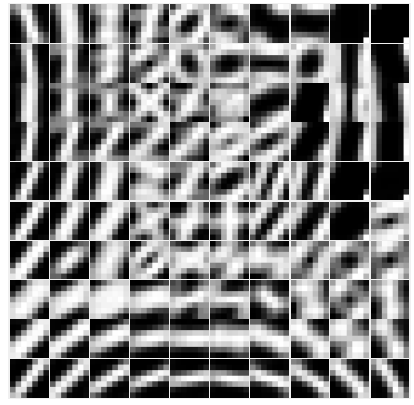

(d)

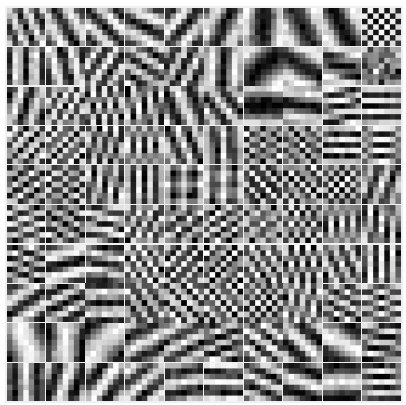

(h)

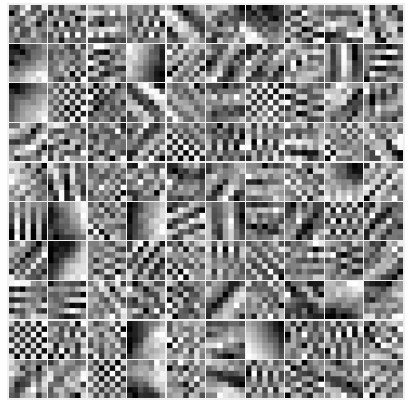

(e)

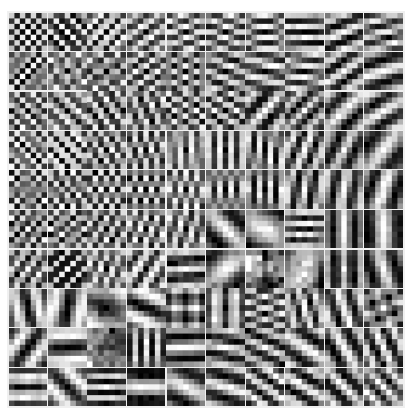

(i)

Figure 4: Different filter banks with filter size 7×7: (a) PCA components, (b) DCT bases, (c) Gabor filters with 5 scales and 8 orientations, (d) SOM weights, (e) randomly selected MRF patterns, (f) MRF-SOM, (g) MRF-SOM TI , (h) MRF Mot5 $^{-S O M}$ and (i) MRF Rot5-SOM $_{\text {TI }}$ features.

Table 6: Classification results and comparison of using different types of filters on MNIST dataset.

\begin{tabular}{|c|c|c|}
\hline Filter Type & \#Filters & Error Rate (\%) \\
\hline$\overline{\mathrm{PCA}}$ & 49 & $0.37 \pm 0.03$ \\
\hline DCT & 49 & $0.34 \pm 0.03$ \\
\hline Gabor & 40 & $0.39 \pm 0.01$ \\
\hline $\mathrm{PCA}+\mathrm{DCT}$ & 98 & $0.34 \pm 0.04$ \\
\hline $\mathrm{PCA}+$ Gabor & 89 & $0.35 \pm 0.03$ \\
\hline DCT + Gabor & 89 & $0.34 \pm 0.04$ \\
\hline $\mathrm{PCA}+\mathrm{DCT}+\mathrm{Gabor}$ & 138 & $0.36 \pm 0.02$ \\
\hline MRF & 49 & $0.43 \pm 0.02$ \\
\hline SOM & 49 & $0.39 \pm 0.02$ \\
\hline MRF-SOM & 49 & $0.45 \pm 0.03$ \\
\hline MRF-SOM & 49 & $0.35 \pm 0.01$ \\
\hline $\mathrm{MRF}_{\mathrm{Rot} 5}$ & 49 & $0.35 \pm 0.03$ \\
\hline $\mathrm{MRF}_{\text {Rot5 }}-\mathrm{SOM}$ & 49 & $0.33 \pm 0.02$ \\
\hline $\mathrm{MRF}_{\text {Rot5}}-\mathrm{SOM}_{\mathrm{TI}}$ & 49 & $0.33 \pm 0.01$ \\
\hline MRF & 100 & $0.40 \pm 0.03$ \\
\hline SOM & 100 & $0.37 \pm 0.02$ \\
\hline MRF-SOM & 100 & $0.41 \pm 0.02$ \\
\hline MRF-SOM $_{\mathrm{TI}}$ & 100 & $0.33 \pm 0.02$ \\
\hline $\mathrm{MRF}_{\mathrm{Rot} 5}$ & 100 & $0.33 \pm 0.02$ \\
\hline $\mathrm{MRF}_{\text {Rot5}}-\mathrm{SOM}$ & 100 & $0.33 \pm 0.02$ \\
\hline $\mathrm{MRF}_{\text {Rot } 5}-\mathrm{SOM}_{\mathrm{TI}}$ & 100 & $\mathbf{0 . 3 2} \pm 0.03$ \\
\hline
\end{tabular}

digits either contain broken or strange strokes, or are ambiguous or have confusing labels. In addition, when ${ }^{715}$ data augmentation (translation and small distortions) was used, the proposed method achieved the state-of-the-art performance of mean error rate $0.24 \%$.

Despite using smaller number of parameters, the proposed method still outperformed the baseline models ${ }^{720}$ with less training time and computational complexity. The 695 rotated MNIST datsets. Results are shown in Table 7 For a fair comparison, we did not include the results of methods that used heavily augmented training samples wo with large distortions or other information. It can be seen that the proposed approach outperformed most of the state-of-the-art models with fewer parameters and less training time.

\subsection{Experiments on CIFAR-10 and CIFAR-100 Datasets}

Data pre-processing strategy was the same for these two datasets. The original $32 \times 32 \mathrm{RGB}$ images were first normalised using data standardisation strategy to have zero mean and unit variance. Then the PCA whitening correlations. No data augmentation was used.

The architecture used started with a prefixed filter bank as the first convolutional layer, followed by a $1 \times 1$ convolutional layer as regularisations and a dropout layer to prevent overfitting. Then two further convolutionpooling-dropout blocks were used, followed by fully connected layers with softmax loss for classification. As shown in Table 8 , for both the CIFAR-10 and CIFAR100 datasets, the networks share the same architecture except for the final output layer that produces probabilities corresponding to different classes. Baseline models for 


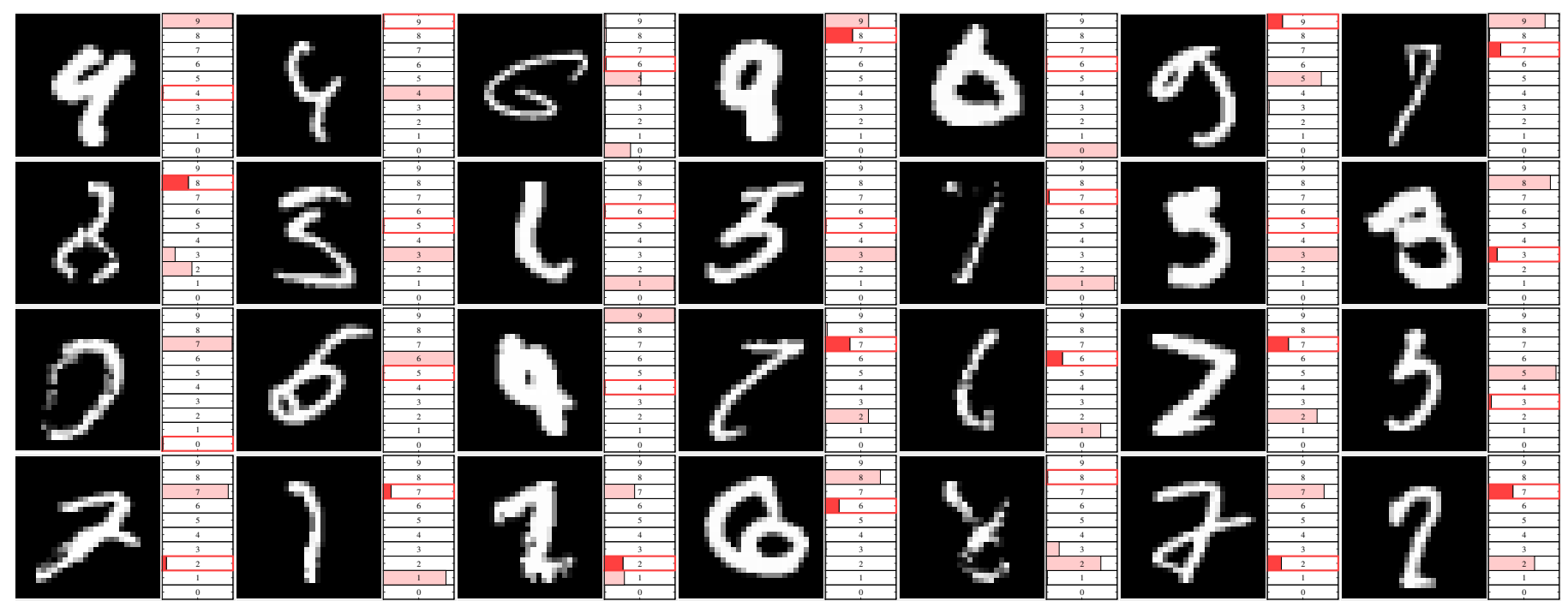

Figure 5: Errors of the MNIST dataset with probabilities of each class. The ground truth labels are marked in red.

Table 7: Comparison of error rate (\%) on MNIST and rotated MNIST datasets.

\begin{tabular}{|c|c|c|c|}
\hline Methods & \#Params & MNIST & $\begin{array}{l}\text { Rotated } \\
\text { MNIST }\end{array}$ \\
\hline \multicolumn{4}{|c|}{ With Data Augmentation } \\
\hline MCDNN 5 & $2.66 \mathrm{M}$ & 0.23 & - \\
\hline DropConnect 8 & - & 0.21 & - \\
\hline APAC 13 & - & 0.23 & - \\
\hline $\mathrm{MRF}_{\mathrm{Rot} 5}-\mathrm{SOM}_{\mathrm{TI}}-\mathrm{CNN}$ & $0.59 \mathrm{M}$ & $0.24 \pm 0.02$ & - \\
\hline \multicolumn{4}{|c|}{ Without Data Augmentation } \\
\hline CNN [4] & $0.23 \mathrm{M}$ & 0.53 & - \\
\hline Stochastic Pooling 6 & $0.2 \mathrm{M}$ & 0.47 & - \\
\hline Maxout [7] & - & 0.45 & - \\
\hline DropConnect 8 & - & 0.57 & - \\
\hline Network in Network 9] & - & 0.47 & - \\
\hline \multicolumn{4}{|l|}{ Fractional Pooling [11] } \\
\hline-1 test & $0.52 \mathrm{M}$ & 0.50 & - \\
\hline-12 tests & $6.21 \mathrm{M}$ & 0.32 & - \\
\hline Deeply Supervised 12 & $0.56 \mathrm{M}$ & 0.39 & - \\
\hline RCNN-32 14 & $0.08 \mathrm{M}$ & 0.42 & - \\
\hline RCNN-64 14 & $0.30 \mathrm{M}$ & 0.32 & - \\
\hline RCNN-96 14 & $0.67 \mathrm{M}$ & 0.31 & - \\
\hline ScatNet 22] & - & 0.43 & - \\
\hline PCANet 23 & - & 0.62 & 7.37 \\
\hline Gabor Assisted CNN 49 & - & 1.53 & - \\
\hline MRF-CNN 18 & $0.59 \mathrm{M}$ & $0.38 \pm 0.01$ & $3.21 \pm 0.04$ \\
\hline Baseline & $0.76 \mathrm{M}$ & $0.31 \pm 0.02$ & $2.70 \pm 0.02$ \\
\hline MRF-SOM-CNN & $0.59 \mathrm{M}$ & $0.41 \pm 0.02$ & $3.12 \pm 0.03$ \\
\hline MRF-SOM $_{\mathrm{TI}}-\mathrm{CNN}$ & $0.59 \mathrm{M}$ & $0.33 \pm 0.02$ & $2.74 \pm 0.07$ \\
\hline $\mathrm{MRF}_{\mathrm{Rot} 5-\mathrm{CNN}}$ & $0.59 \mathrm{M}$ & $0.34 \pm 0.02$ & $2.69 \pm 0.05$ \\
\hline $\mathrm{MRF}_{\mathrm{Rot} 5}-\mathrm{SOM}-\mathrm{CNN}$ & $0.59 \mathrm{M}$ & $0.32 \pm 0.01$ & $2.69 \pm 0.06$ \\
\hline $\mathrm{MRF}_{\mathrm{Rot} 5}-\mathrm{SOM}_{\mathrm{TI}}-\mathrm{CNN}$ & $0.59 \mathrm{M}$ & $\mathbf{0 . 3 0} \pm 0.02$ & $\mathbf{2 . 6 8} \pm 0.02$ \\
\hline
\end{tabular}

these two datasets were standard $\mathrm{CNNs}_{\mathrm{s}}$ and all layers were $\mathrm{e}_{70}$ trained in supervised mode.

\subsubsection{Comparisions and Discussion}

Based on the results of Table 4, for the CIFAR-10 and CIFRA-100 datasets, the optimal filter size was $3 \times 3.745$ Therefore, the networks were trained on $3 \times 3$ filter banks containing $50 \times 50 \mathrm{MRF}_{\text {Rot } 5}-\mathrm{SOM}_{\mathrm{TI}}$ clustered filters. The
Table 8: Architecture used on CIFAR-10 and CIFAR-100 datasets.

\begin{tabular}{c}
\hline Input $32 \times 32$ RGB Images \\
\hline$q \times q$ conv. $d^{2}$ ReLU with zero padding $(q-1) / 2$ \\
$1 \times 1$ conv. 192 ReLU \\
\hline Dropout prob $=0.5$ \\
$3 \times 3$ conv. 192 ReLU \\
$3 \times 3$ conv. 192 ReLU \\
$3 \times 3$ max-pooling stride 2 \\
\hline Dropout, prob $=0.5$ \\
\hline $3 \times 3$ conv. 192 ReLU \\
$3 \times 3$ conv. 192 ReLU \\
$3 \times 3$ max-pooling stride 2 \\
\hline Dropout, prob $=0.5$ \\
\hline $3 \times 3$ conv. 192 ReLU \\
$3 \times 3$ conv. 192 ReLU \\
\hline Dropout, prob $=0.5$ \\
$1 \times 1$ conv. 96 ReLU \\
$1 \times 1$ conv. 10 ReLU \\
\hline 10 -class or 100 -class softmax
\end{tabular}

classification results are given in Table 9 along with that of some state-of-the-art methods. With similar numbers of parameters, the proposed $\mathrm{MRF}_{\mathrm{Rot5}}-\mathrm{SOM}_{\mathrm{TI}^{-}}$ CNN outperformed baseline models on both CIFAR-10 and CIFAR-100 datasets. Compared to other state-ofthe-art approaches, the incorporation of the $\mathrm{MRF}_{\text {Rot5- }}$ $\mathrm{SOM}_{\mathrm{TI}}$ features achieved remarkable results without adding complex layers, altering cost functions, or changing the gradient descent algorithm. It was only outperformed by much complicated networks such as the RCNN-160 14] (hard to train deeper models due to many reasons such as vanishing and exploding gradients [72, 73]), the ELU-Network [15] (with 18 convolutional layers and 165000 iterations), ResNet 19] (with 1202 convolutional layers and 19.4M parameters), WRN [16] (deeper and wider architectures with large number of parameters) and DenseNet-BC [17] (with over 100 convolutional depth and much larger amount of parameters), which used augmented data and more sophisticated networks 
Table 9: Comparison of error rate (\%) on CIFAR-10 and CIFAR-100 datasets.

\begin{tabular}{|c|c|c|c|c|c|c|c|}
\hline Methods & \#Params & CIFAR-10 & CIFAR-100 & Methods & \#Params & CIFAR-10 & CIFAR-100 \\
\hline \multicolumn{4}{|c|}{ Without Data Augmentation } & \multicolumn{4}{|c|}{ With Data Augmentation } \\
\hline Stochastic Pooling [6] & $0.2 \mathrm{M}$ & 15.13 & 42.51 & MCDNN [5] & $1.7 \mathrm{M}$ & 11.21 & - \\
\hline Maxout 7] & $6 \mathrm{M}$ & 11.68 & 34.54 & Maxout [7] & $6 \mathrm{M}$ & 9.38 & - \\
\hline DropConnect [8] & - & 18.70 & - & DropConnect 8 & - & 9.32 & - \\
\hline Network in Network 9] & $1.0 \mathrm{M}$ & 10.41 & 35.68 & Network in Network 9] & $1.0 \mathrm{M}$ & 8.81 & - \\
\hline All-CNN 10] & $1.4 \mathrm{M}$ & 9.08 & 33.71 & All-CNN [10] & $1.4 \mathrm{M}$ & 7.25 & - \\
\hline Deeply Supervised 12 & $1.0 \mathrm{M}$ & 9.69 & 34.57 & Deeply Supervised [12] & $1.0 \mathrm{M}$ & 7.97 & - \\
\hline RCNN-96 14 & $0.67 \mathrm{M}$ & 9.31 & 34.18 & RCNN-96 14 & 0.67 & 7.37 & - \\
\hline RCNN-160 14 & $1.86 \mathrm{M}$ & 8.69 & 31.75 & RCNN-160 14 & $1.86 \mathrm{M}$ & 7.09 & - \\
\hline MRF-CNN 18 & $2.5 \mathrm{M}$ & $8.75 \pm 0.14$ & $33.92 \pm 0.17$ & APAC 13 & - & 10.33 & - \\
\hline Baseline & $2.6 \mathrm{M}$ & $8.18 \pm 0.07$ & $33.07 \pm 0.08$ & Scat+WRN-16-8 48 & 11.0M & 6.90 & - \\
\hline MRF-SOM-CNN & $2.5 \mathrm{M}$ & $10.12 \pm 0.15$ & $37.06 \pm 0.19$ & ResNet-110 19 & $1.7 \mathrm{M}$ & $6.61 \pm 0.16$ & - \\
\hline MRF-SOM ${ }_{\mathrm{TI}}-\mathrm{CNN}$ & $2.5 \mathrm{M}$ & $8.49 \pm 0.12$ & $33.68 \pm 0.11$ & ResNet-1202 19] & $19.4 \mathrm{M}$ & 7.93 & - \\
\hline $\mathrm{MRF}_{\mathrm{Rot} 5}-\mathrm{CNN}$ & $2.5 \mathrm{M}$ & $8.67 \pm 0.10$ & $33.91 \pm 0.22$ & PreAct-ResNet-110 [20] & $1.7 \mathrm{M}$ & 6.37 & 24.71 \\
\hline $\mathrm{MRF}_{\text {Rot5}}-\mathrm{SOM}-\mathrm{CNN}$ & $2.5 \mathrm{M}$ & $9.51 \pm 0.11$ & $36.60 \pm 0.21$ & $\mathrm{MRF}_{\text {Rot5}}-\mathrm{SOM}_{\mathrm{TI}}$ & $1.7 \mathrm{M}$ & $\mathbf{5 . 9 8} \pm 0.15$ & $\mathbf{2 4 . 3 1} \pm 0.29$ \\
\hline $\mathrm{MRF}_{\text {Rot5}}-\mathrm{SOM}_{\mathrm{TI}}-\mathrm{CNN}$ & $2.5 \mathrm{M}$ & $\mathbf{8 . 1 0} \pm 0.11$ & $32.98 \pm 0.18$ & +PreAct-ResNet-110 & & & \\
\hline
\end{tabular}

architectures with much more parameters.

Furthermore, we also incorporated the proposed

$\mathrm{MRF}_{\text {Rot5-SOM }} \mathrm{SO}_{\mathrm{TI}}$ features with PreAct-ResNet-110 [20. The first convolutional layer in the PreAct-ResNet-110 was replaced by the $\mathrm{MRF}_{\mathrm{Rot} 5}-\mathrm{SOM}_{\mathrm{TI}}$ based filters (SOM size: $10 \times 10$ ) followed by a $1 \times 1$ convolutional layer to compress790 the feature maps. The remaining layers stayed unchanged with the same hyperparameters, initialisations and data augmentation strategy. Compared to the original PreActResNet-110, the combination of $\mathrm{MRF}_{\text {Rot5 }}-\mathrm{SOM}_{\mathrm{TI}}$ features with ResNet architecture achieves better performance with 795 comparable number of parameters, as shown in Table 9 .

\section{Conclusions}

This paper presents an approach to feature learning by combining Markov random field (MRF) models with $\mathrm{w}_{800}$ self-organising maps (SOMs) to generate various generic feature descriptors. These features can be incorporated as low-level features in convolutional neural networks (CNNs), which are then trained to learn only implicit high- ${ }_{805}$ level features. By choosing different potential functions for the MRF models, a broad range of spatial patterns are generated. Convolutional translation-invariant SOMs are then trained on these patterns to derive the efficient ${ }_{810}$ representative filters. These filters extract generic salient features invariant to translation, small rotation and distortion. We examined such feature learning mechanism and compared with the existing methods in image classification ${ }_{815}$ tasks as well as investigated impact of feature extraction in the framework of CNNs.

The results have confirmed the effectiveness of the proposed approach with performance on several image 820 datasets among the best in the comparison, only exceeded by either extremely larger networks or those trained with excessive data-augmentation. The proposed method not only combines respective strengths of MRFs and825 SOMs to model both intra- and inter-image dependencies, but also integrates the expressive power of CNNs with the probabilistic texture models. The data-independent feature learning mechanism can produce efficient, generic and transferable features for various tasks, as well as can gain better understanding of image representations, particularly in early layers of CNNs. Whilst some previous literature has shown that existing (learned) lowlevel features do not generalise well 23] 25, this work has demonstrated that generic image features exist and can be learned independently. It also demonstrated the usefulness of MRFs and unsupervised learning in deriving and understanding such fundamental image features.

\section{References}

[1] Y. Bengio, A. Courville, P. Vincent, Representation learning: A review and new perspectives, IEEE Trans. Pattern Anal. Mach. Intell. 35 (8) (2013) 1798-1828.

[2] G. E. Hinton, S. Osindero, Y.-W. Teh, A fast learning algorithm for deep belief nets, Neural Comput. 18 (7) (2006) 1527-1554.

[3] Y. LeCun, Y. Bengio, G. E. Hinton, Deep learning, Nature 521 (7553) (2015) 436-444.

[4] K. Jarrett, K. Kavukcuoglu, Y. LeCun, et al., What is the best multi-stage architecture for object recognition?, in: Proc. IEEE Int. Conf. Comput. Vis. (ICCV), 2009, pp. 2146-2153.

[5] D. Cireşan, U. Meier, J. Schmidhuber, Multi-column deep neural networks for image classification, in: Proc. IEEE Conf. Comput. Vis. Pattern Recognit. (CVPR), 2012, pp. 3642-3649.

[6] M. D. Zeiler, R. Fergus, Stochastic pooling for regularization of deep convolutional neural networks, arXiv preprint arXiv:1301.3557.

[7] I. J. Goodfellow, D. Warde-Farley, M. Mirza, A. Courville, Y. Bengio, Maxout networks, arXiv preprint arXiv:1302.4389.

[8] L. Wan, M. Zeiler, S. Zhang, Y. L. Cun, R. Fergus, Regularization of neural networks using DropConnect, in: Proc. Int. Conf. Mach. Learn. (ICML), 2013, pp. 1058-1066.

[9] M. Lin, Q. Chen, S. Yan, Network in network, arXiv preprint arXiv:1312.4400.

[10] J. T. Springenberg, A. Dosovitskiy, T. Brox, M. Riedmiller, Striving for simplicity: The all convolutional net, arXiv preprint arXiv:1412.6806.

[11] B. Graham, Fractional max-pooling, arXiv preprint arXiv:1412.6071.

[12] C.-Y. Lee, S. Xie, P. Gallagher, Z. Zhang, Z. Tu, Deeplysupervised nets, in: Proc. Int. Conf. Artif. Intell. Stats. (AISTATS), 2015, pp. 562-570. 
[13] I. Sato, H. Nishimura, K. Yokoi, APAC: Augmented pattern classification with neural networks, arXiv preprint900 arXiv:1505.03229.

[14] M. Liang, X. Hu, Recurrent convolutional neural network for object recognition, in: Proc. IEEE Conf. Comput. Vis. Pattern Recognit. (CVPR), 2015, pp. 3367-3375.

[15] D.-A. Clevert, T. Unterthiner, S. Hochreiter, Fast and accurate905 deep network learning by exponential linear units (ELUs), arXiv preprint arXiv:1511.07289.

[16] S. Zagoruyko, N. Komodakis, Wide residual networks, arXiv preprint arXiv:1605.07146.

[17] G. Huang, Z. Liu, K. Q. Weinberger, L. van der Maaten,910 Densely connected convolutional networks, in: Proc. IEEE Conf. Comput. Vis. Pattern Recognit. (CVPR), Vol. 1, 2017, p. 3.

[18] Y. Peng, H. Yin, Markov random field based convolutional neural networks for image classification, in: Proc. Int. Conf.915 Intell. Data Eng. Autom. Learn. (IDEAL), 2017, pp. 387-396.

[19] K. He, X. Zhang, S. Ren, J. Sun, Deep residual learning for image recognition, in: Proc. IEEE Conf. Comput. Vis. Pattern Recognit. (CVPR), 2016, pp. 770-778.

[20] K. He, X. Zhang, S. Ren, J. Sun, Identity mappings in deep920 residual networks, in: Proc. IEEE Eur. Conf. Comput. Vis. (ECCV), 2016, pp. 630-645.

[21] Y. LeCun, K. Kavukcuoglu, C. Farabet, Convolutional networks and applications in vision, in: Proc. IEEE Int. Symp. Circuits Syst. (ISCAS), 2010, pp. 253-256.

[22] J. Bruna, S. Mallat, Invariant scattering convolution networks, IEEE Trans. Pattern Anal. Mach. Intell. 35 (8) (2013) 18721886 .

[23] T.-H. Chan, K. Jia, S. Gao, J. Lu, Z. Zeng, Y. Ma, PCANet: A simple deep learning baseline for image classification?, IEEE930 Trans. Image Process. 24 (12) (2015) 5017-5032.

[24] C.-Y. Low, A. B.-J. Teoh, C.-J. Ng, Multi-fold Gabor, PCA and ICA filter convolution descriptor for face recognition, IEEE Trans. Circuits Syst. Video Technol.

[25] C. J. Ng, A. B. J. Teoh, DCTNet: A simple learning-freeg35 approach for face recognition, in: Proc. Annu. Summit Asia Pac. Signal Inf. Process. Assoc. (APSIPA), 2015, pp. 761-768.

[26] L. Dong, L. He, M. Mao, G. Kong, X. Wu, Q. Zhang, X. Cao, E. Izquierdo, CUNet: A compact unsupervised network for image classification, IEEE Trans. Multimedia.

27] J. Yosinski, J. Clune, Y. Bengio, H. Lipson, How transferable are features in deep neural networks?, in: Proc. Conf. Adv. Neural Inf. Process. Syst. (NIPS), 2014, pp. 3320-3328.

[28] L. Fei-Fei, R. Fergus, P. Perona, One-shot learning of object categories, IEEE Trans. Pattern Anal. Mach. Intell. 28 (4)945 (2006) 594-611.

[29] M. Palatucci, D. Pomerleau, G. E. Hinton, T. M. Mitchell, Zeroshot learning with semantic output codes, in: Proc. Conf. Adv. Neural Inf. Process. Syst. (NIPS), 2009, pp. 1410-1418.

[30] B. Julesz, Visual pattern discrimination, IRE Trans. Inf. Theory950 8 (2) (1962) 84-92.

[31] B. Julesz, Textons, the elements of texture perception, and their interactions, Nature 290 (5802) (1981) 91-97.

[32] S. Z. Li, A Markov random field model for object matching under contextual constraints, in: Proc. IEEE Conf. Comput.955 Vis. Pattern Recognit. (CVPR), 1994, pp. 866-866.

[33] S. Z. Li, Markov random field modelling in image analysis, Springer Science and Business Media, 2009.

[34] S. Geman, D. Geman, Stochastic relaxation, Gibbs distributions, and the Bayesian restoration of images., IEEE Trans.960 Pattern Anal. Mach. Intell. 6 (6) (1984) 721-741.

[35] T. Kohonen, Self-organized formation of topologically correct feature maps, Biol. Cybern. 43 (1) (1982) 59-69.

[36] Y. LeCun, L. Bottou, Y. Bengio, P. Haffner, Gradient-based learning applied to document recognition, Proc. IEEE 86 (11)965 (1998) 2278-2324.

[37] H. Larochelle, D. Erhan, A. Courville, J. Bergstra, Y. Bengio, An empirical evaluation of deep architectures on problems with many factors of variation, in: Proc. Int. Conf. Mach. Learn.
(ICML), 2007, pp. 473-480.

[38] A. Krizhevsky, G. Hinton, Learning multiple layers of features from tiny images.

[39] D. G. Lowe, Object recognition from local scale-invariant features, in: Proc. IEEE Int. Conf. Comput. Vis. (ICCV), Vol. 2, 1999, pp. 1150-1157.

[40] W. T. Freeman, M. Roth, Orientation histograms for hand gesture recognition, in: Proc. IEEE Int. Conf. Autom. Face Gesture Recognit. (FG), Vol. 12, 1995, pp. 296-301.

[41] T. Ojala, M. Pietikainen, T. Maenpaa, Multiresolution grayscale and rotation invariant texture classification with local binary patterns, IEEE Trans. Pattern Anal. Mach. Intell. 24 (7) (2002) 971-987.

[42] W. Huang, H. Yin, Robust face recognition with structural binary gradient patterns, Pattern Recognit. 68 (2017) 126-140.

[43] A. Krizhevsky, I. Sutskever, G. E. Hinton, ImageNet classification with deep convolutional neural networks, in: Proc. Conf. Adv. Neural Inf. Process. Syst. (NIPS), 2012, pp. 1097-1105.

44] K. Simonyan, A. Zisserman, Very deep convolutional networks for large-scale image recognition, arXiv preprint arXiv:1409.1556.

[45] W. Samek, A. Binder, G. Montavon, S. Lapuschkin, K.-R. Müller, Evaluating the visualization of what a deep neural network has learned, IEEE Trans. Neural Netw. Learn. Syst. 28 (11) (2017) 2660-2673.

[46] M. D. Zeiler, R. Fergus, Visualizing and understanding convolutional networks, in: Proc. IEEE Eur. Conf. Comput. Vis. (ECCV), 2014, pp. 818-833.

[47] J. Yosinski, J. Clune, A. Nguyen, T. Fuchs, H. Lipson, Understanding neural networks through deep visualization, arXiv preprint arXiv:1506.06579.

[48] E. Oyallon, E. Belilovsky, S. Zagoruyko, Scaling the scattering transform: Deep hybrid networks, in: Proc. IEEE Int. Conf. Comput. Vis. (ICCV), 2017.

[49] S. S. Sarwar, P. Panda, K. Roy, Gabor filter assisted energy efficient fast learning convolutional neural networks, in: Proc. IEEE Int. Symp. Low Power Electron. Des. (ISLPED), 2017, pp. 1-6.

[50] S. Mehrkanoon, J. A. Suykens, Deep hybrid neural-kernel networks using random Fourier features, Neurocomputing 298 (2018) 46-54.

[51] K. Pearson, On lines and planes of closest fit to systems of points in space, Lond. Edinb. Dubl. Phil. Mag. 2 (11) (1901) 559-572.

[52] H. Hotelling, Analysis of a complex of statistical variables into principal components., J. Educ. Psychol. 24 (7) (1933) 498-520.

[53] N. Ahmed, T. Natarajan, K. R. Rao, Discrete cosine transform, IEEE Trans. Comput. 100 (1) (1974) 90-93.

[54] J. G. Daugman, Uncertainty relation for resolution in space, spatial frequency, and orientation optimized by two-dimensional visual cortical filters, J. Opt. Soc. Amer. A. 2 (7) (1985) 11601169 .

[55] S. Mardělja, Mathematical description of the responses of simple cortical cells, J. Opt. Soc. Amer. 70 (11).

[56] J. Besag, Spatial interaction and the statistical analysis of lattice systems, J. R. Stat. Soc. Series B Stat. Methodol. (1974) $192-236$

[57] J. M. Hammersley, P. E. Clifford, Markov random fields on finite graphs and lattices, Unpublished manuscript.

[58] P. Clifford, Markov random fields in statistics, Disorder in physical systems: A volume in honour of John M. Hammersley 19

[59] G. R. Cross, A. K. Jain, Markov random field texture models, IEEE Trans. Pattern Anal. Mach. Intell. 5 (1) (1983) 25-39.

[60] H. Elliott, H. Derin, R. Cristi, D. Geman, Application of the gibbs distribution to image segmentation, in: Proc. IEEE Int. Conf. Acoust. Speech Signal Process. (ICASSP), Vol. 9, 1984, pp. $678-681$.

[61] H. Derin, H. Elliott, Modeling and segmentation of noisy and textured images using gibbs random fields, IEEE Trans. Pattern Anal. Mach. Intell. (1) (1987) 39-55.

[62] R. Kashyap, R. Chellappa, Estimation and choice of neighbors 
in spatial-interaction models of images, IEEE Trans. Image Process. 29 (1) (1983) 60-72.

[63] S. C. Dass, Markov random field models for directional field and singularity extraction in fingerprint images, IEEE Trans. Image Process. 13 (10) (2004) 1358-1367.

[64] N. Metropolis, A. W. Rosenbluth, M. N. Rosenbluth, A. H. Teller, E. Teller, Equation of state calculations by fast computing machines, J. Chem. Phys. 21 (6) (1953) 1087-1092.

[65] J. M. Hammersley, D. C. Handscomb, Monte Carlo methods, London: Methuen and Company, 1964.

[66] T. Kohonen, Self-organisation and associative memory, Springer-Verlag, 1984.

[67] M. Cottrell, J. Fort, A stochastic model of retinotopy: A self organizing process, Biol. Cybern. 53 (6) (1986) 405-411.

[68] E. Erwin, K. Obermayer, K. Schulten, Self-organizing maps: ordering, convergence properties and energy functions, Biol. Cybern. 67 (1) (1992) 47-55.

[69] H. Yin, N. M. Allinson, On the distribution and convergence of feature space in self-organizing maps, Neural Comput. 7 (6) (1995) 1178-1187.

990 [70] H. Yin, The self-organizing maps: Background, theories, extensions and applications, in: Computational Intelligence: A Compendium, Springer, 2008, pp. 715-762.

[71] F. Blayo, Kohonen self-organizing maps: Is the normalization necessary?, Complex Syst. 6 (6) (1992) 105-123.

995 [72] Y. Bengio, P. Simard, P. Frasconi, et al., Learning long-term dependencies with gradient descent is difficult, IEEE Trans. Neural Netw. 5 (2) (1994) 157-166.

[73] R. Pascanu, T. Mikolov, Y. Bengio, On the difficulty of training recurrent neural networks, in: Proc. Int. Conf. Mach. Learn. (ICML), 2013, pp. 1310-1318. 